\title{
LA SEMIÓTICA DE LA COMUNICACIÓN MASIVA EN ESPAÑA: SUS AVATARES ACADÉMICOS CONTADOS POR SUS PROTAGONISTAS
}

\author{
MASS MEDIA SEMIOTICS IN SPAIN: \\ ITS UPS AND DOWNS AS RECALLED BY ITS PROTAGONISTS
}

\author{
Luis Pablo FRANCESCUTTI PÉREZ \\ Universidad Rey Juan Carlos \\ luispablo.francescutti@urjc.es
}

Resumen: La comunicación masiva ha sido y es un objeto privilegiado de la semiótica. A partir del testimonio de seis semiólogos españoles en este artículo se reconstruye la recepción y aclimatación de esa línea de estudios en España. Sus evocaciones parten de la llegada de la disciplina a mediados de los años ' 60 , continúan con su período de moda intelectual (años '70) y con su inserción en las facultades de comunicación (años '80), y concluyen en la situación actual, señalada por la falta de relevo generacional, el avance de paradigmas empírico-positivistas y las dudas sobre su continuidad académica.

Palabras clave: Semiótica. Comunicación masiva. Trayectoria académica. España.

\begin{abstract}
Mass communication is a prominent field of study for semiotics. In this article the Spanish reception and institutionalization of this branch of semiotics is reconstructed. The data collected from the interviews with six distinguished Spanish semioticians allow following the process since its introduction in the mid Sixties to a formative phase in the Seventies, followed by its institutionalisation in the Eighties. These conversational narratives end at the current situation, where the difficulties for generational renewal and the ascendancy of empiricist methodologies and positivist paradigms raise serious doubts about the future of the sub-discipline.
\end{abstract}


Key Words: Semiotics. Mass communication. Academic history. Spain.

\section{INTRODUCCIÓN}

La semiótica, la ciencia de los signos, cristaliza como un saber diferenciado a mediados del siglo XX y cobra entidad institucional a finales de los años '60, con la formación de la International Association for Semiotic Studies (IASS), la creación de asociaciones nacionales, la realización de congresos y la aparición de revistas y colecciones editoriales especializadas. Tiene sus epicentros en Italia, en torno a la figura de Umberto Eco; en Francia, con eje en Roland Barthes y Algirdas Greimas; en Estados Unidos, en torno al magisterio de Thomas Sebeok; en la Unión Soviética, liderada por la Escuela de Tartu; y en América Latina, en la senda abierta por Eliseo Verón.

España se fue acoplando a esa tendencia a lo largo de la década de los años '70. Bobes Naves (1973), Urrutia (1975), Abril (1979), Romera Castillo (1980) y Serrano (1981), entre otros, introducen la emergente disciplina al público autóctono. A partir de la década de 1980 va cuajando una comunidad de investigadores que aplican la semiótica a los más variados objetos.

Si la existencia de asociaciones y publicaciones es un parámetro del asentamiento de una disciplina, puede afirmarse que la semiótica toma carta de ciudadanía en España en 1983, con la creación de la Asociación Española de Semiótica (AES), por iniciativa del profesor José Romera Castillo (http://www.aesemiotica.es/congresos [20/02/2019]) y de la Asociación de Estudios Semióticos de Barcelona; seguidas de la Asociación Andaluza de Semiótica, en 1985; la Asociación Vasca de Semiótica, en 1988; y la Asociación Gallega de Semiótica, en 1993 (Romera Castillo, 1991).

Otro indicador de su arraigo lo proveen las publicaciones especializadas: Estudios Semióticos, editada entre 1983 y 1988 por la Asociación de Estudios Semióticos de Barcelona; Discurso, publicada entre 1987 y 2004 por la Asociación Andaluza de Semiótica; Semiosfera, de la Universidad Carlos III, que en su primera época (1994-98) hace eje en la semiótica; Entretextos, publicada entre 2003 y 2010 en la Universidad 
de Granada, con foco en la Escuela de Tartu. Entre todas, es preciso destacar Signa. Revista de la Asociación Española de Semiótica (https:// dialnet.unirioja.es/servlet/revista? codigo $=1349$ [20/02/2019], creada y dirigida por Romera Castillo (2016), dentro del Centro de Investigación de Semiótica Literaria, Teatral y Nuevas Tecnologías (https://www2. uned.es/centro-investigacion-SELITEN@T/index2.html][20/02/2019]), que también dirige el mencionado investigador y que es el órgano de la AES desde 1991 y hoy la única publicación consagrada a la disciplina en España. A ello se añaden iniciativas editoriales como las colecciones Semiótica y Crítica (Ed. Alfar), coordinada por Manuel Ángel Vázquez Medel, y Signo e Imagen (Cátedra), supervisada por Jenaro Talens.

Eso en cuanto a la semiótica española en su totalidad, como ha estudiado Romera Castillo en una serie numerosa de trabajos. Ahora bien, en el vasto campo de los estudios semióticos los fenómenos de la comunicación masiva, sobre los que se centrará nuestra atención exclusivamente ahora, han ocupado una posición sobresaliente. Lo acreditan los trabajos sobre la publicidad y la cultura de masas de Barthes $(1980,1974)$, de Eco sobre el cómic y el folletín (1978) y la neotelevisión (1983); o el de Verón relativo al acontecimiento periodístico (1983). El presente artículo versa sobre esta área de estudios, cuya inserción académica en estas tierras no ha sido estudiada más que tangencialmente en los diversos trabajos referidos a la implantación general de la disciplina en el Estado español (Romera Castillo, 1991 y 2015; Yllera, 1979; Pozuelo-Yvancos, 1999; Ríos, 2015), en Cataluña (Velázquez y Lacalle, 1999), en Andalucía (Vázquez Medel, 1999) y en Galicia (Paz Gago y Couto Cantero, 1999).

Colmar en parte esa laguna es el propósito de esta investigación diseñada en el marco de un proyecto de cartografiado de las corrientes teóricas que modelaron los estudios en comunicación en España ${ }^{1}$. Para ello nos basaremos en el testimonio de semiólogos que tuvieron una participación relevante en la promoción de esta área de la semiótica en los últimos cuarenta años.

1“25 años de investigación sobre Comunicación en España (1990-2015): producción científica, comunidad académica y contexto institucional", Plan Estatal de I+D+i (CSO2013-40684-P). 


\section{OBJETIVOS}

Partiendo de lo expuesto, nos hemos fijado objetivos que van de lo particular a lo general. En el plano más concreto y local, intentaremos reconstruir la llegada de la semiótica de la comunicación de masas a las universidades españolas, las escuelas extranjeras que definieron su orientación inicial, sus principales objetos de estudio y su institucionalización en las facultades de periodismo y/o ciencias de la comunicación, tal como la vivieran sus protagonistas.

En un plano general, aspiramos a que el relato de la experiencia de los semiólogos españoles enriquezca la comprensión del proceso de difusión y recepción internacional de la semiótica, y en especial de los escollos con los que, a su modo de ver, tropezó su aclimatación.

\section{METODOLOGÍA}

La pesquisa se apoya en entrevistas en profundidad realizadas a seis profesores de universidad con una trayectoria relevante en la semiótica de la comunicación masiva., a quienes se les pidió que, al modo de la historia oral, relacionasen sus carreras con los avatares de su especialidad.

La historia oral es una alternativa pertinente cuando escasean las fuentes documentales y se tiene acceso a quienes vivieron directamente el fenómeno a estudiar. Concebida para dar voz a los sin voz (miembros de colectivos subalternos ausentes en los registros documentales), se ha extendido a otras clases de sujetos. Un ejemplo lo ofrece la historia oral de la ciencia a partir de las narrativas conversacionales de científicos (Weiner, 1988). Como explica Doel (2003), se recurrió a esta metodología al tomarse conciencia de las carencias de los documentos escritos y de la 4 necesidad de ahondar en la ideología de los actores, su visión de su disciplina, y la relación entre los hechos conocidos y la vida personal (ej. la discriminación de género sufrida por las investigadoras).

Desde luego, las fuentes orales no se hallan exentas de deficiencias epistemológicas: errores de datación, fallos de la memoria, o distorsiones más o menos inconscientes del recuerdo como la ilusión biográfica². Por

${ }^{2}$ Este concepto acuñado por Bourdieu (1989) se refiere a la distorsión que los individuos (y sus biógrafos) realizan retrospectivamente de sus vidas al presentarlas como el despliegue resuelto, 
esta razón, quien las utilice deberá cotejar sus hallazgos con otras fuentes (Mariezkurrena Iturmendi, 2008) atento a las posibles discrepancias significativas.

En cuanto a la selección de nuestros entrevistados, nos guiamos por los siguientes criterios: 1) una dilatada carrera universitaria; 2) dedicación plena a la semiótica de la comunicación masiva, al menos durante un periodo de su carrera; y 3 ) adscripción a distintas universidades españolas.

De tal modo fueron seleccionados seis especialistas: Miquel de Moragas, catedrático emérito de la Universidad Autónoma de Barcelona (UAB); Charo Lacalle, catedrática del mismo centro educativo, al igual que José Manuel Pérez Tornero; Jorge Lozano, catedrático de la Universidad Complutense de Madrid (UCM); el ya citado Talens, catedrático de la Universidad de Valencia (UV); y Santos Zunzunegui, catedrático de la Universidad del País Vasco (UPV).

Excepto Moragas, que se licenció en la Universidad de Barcelona, y Talens, que lo hizo en Granada, los demás desarrollaron sus carreras en la universidad donde se graduaron. Uno de los entrevistados se inició en la docencia a finales de los años ' 60 ; tres en los años ' 70 ; y los dos restantes en los ' 80 . Lacalle, Lozano, Talens y Zunzunegui han ejercitado la semiótica desde el inicio de sus trayectorias profesionales, y Moragas y Pérez Tornero solo lo hicieron en los primeros años de su carrera, coincidiendo con el despegue de la disciplina en España (la presencia de tres docentes de la UAB se explica por el papel señero de ese centro en la semiótica española en el ámbito que estudiamos).

Dentro del vasto territorio de la comunicación de masas, cada uno de ellos aplicó sus opciones metodológicas en objetos específicos: Lozano encaró el discurso periodístico mediante el análisis del discurso y, posteriormente adoptó la semiótica de la cultura de la Escuela de Tartu para estudiar la moda y el acontecimiento periodístico. Pérez Tornero dedicó sus conocimientos de la semiótica estructuralista al estudio de la publicidad y la imagen audiovisual; Moragas combinó las perspectivas sociológicas y semióticas en sus trabajos sobre los mass media, la pornografía y la prensa. Talens, se apoyó en su formación en semiótica literaria para analizar el grafitti y los aspectos documentales y ficcionales de la cultura de masas;

rectilíneo y teleológico de un plan perfectamente diseñado, omitiendo la intervención del azar, los tanteos, los rodeos, las apuestas fallidas, los cambios de orientación, etc. 
Lacalle, por su parte, combinó la teoría de Greimas y las aportaciones de Eco en su abordaje de los discursos televisivos (informativos, reality shows, teleseries...); mientras que Zunzunegui aplicó las pautas de la semiótica de cuño greimasiano a la indagación de la imagen fotográfica y cinematográfica.

A lo largo de entrevistas de una hora y media de duración, se les pidió que reconstruyeran sus itinerarios académicos y los conectasen con los estudios semióticos de los mass media y con su implantación universitaria5. Los datos producidos por el análisis de estos relatos fueron cotejados entre sí a fin de detectar coincidencias y discrepancias, y también con las cronologías referidas en los memoriales citados. Finalmente, los resultados se agruparon conforme a criterios cronológicos, diferenciando las etapas cubiertas desde su recepción hasta su estado actual.

\section{ANÁLISIS DE LAS ENTREVISTAS}

\subsection{Apuntes sobre la recepción de la semiótica en España}

Sin excepción, los entrevistados remontan el impacto de la semiótica en España a mediados de los años '60. Moragas, en concreto, lo sitúa en la visita de Eco a Barcelona, en 1966. No por casualidad será en esta ciudad desde donde se difundirá la obra del semiólogo italiano y donde primero se incorporará la semiótica a la universidad, inicialmente en las áreas de diseño y arquitectura:

Profesores como De Ventós, críticos de arte como Cirici Pellicer que descubren en el análisis semiótico una alternativa a las teorias historicistas en arquitectura (...) la primera actividad que recuerdo en España sobre semiótica es el Simposio sobre Semiótica y Arquitectura en Castelldefells (...) con Oriol Bohigas hablando de semiótica de la recepción y Estética (Moragas, comunicación personal, 11 diciembre 2016).

Otra muestra de la receptividad local a la semiótica la ofrece, poco más tarde, la labor pionera de Roman Gubern³ (1974, 1977), a buen seguro

${ }^{3}$ Gubern haría carrera académica en la UAB, donde alcanzaría la posición de catedrático de 
el primer intelectual español que abordó la cultura de masas desde una perspectiva claramente influenciada por la ciencia de los signos: "sin ser un semiólogo, Gubern aplicaba por libre miradas semióticas al cómic, al cine y a la publicidad para averiguar qué significaban un tebeo o una película" (Lozano, comunicación personal, 24 abril 2016).

En la misma época, las carreras de filología, como ha estudiado el profesor Romera Castillo (1988) en los ámbitos literarios y teatrales, también acusaron recibo del pensamiento semiótico y de los precedentes intelectuales que fomentaron en los estudiantes una sensibilidad propicia a su recepción. Talens lo recuerda de la siguiente manera:

A finales de los años '60, los profesores de la universidad de Granada ya hablaban de Todorov y los formalistas rusos, de Tinianov y Shklovski. Recuerdo las clases de Literatura Comparada Medieval de Soria Ortega. No eran estrictamente semiólogos, pero estaban al tanto de su saber (Talens, comunicación personal, 9 marzo 2016).

Dichas resonancias se explican en buena medida por las circunstancias de la época. España vive una gran efervescencia social y cultural, existe un poderoso impulso por salir del aislamiento franquista y ponerse al día con las corrientes de pensamiento más prestigiosas en el contexto internacional. La semiótica se beneficia de esa avidez y deviene moda intelectual:

La semiótica estaba de moda. Resultaba imposible decir nada en el conjunto de las ciencias sociales sin utilizar lajerga y el metalenguaje de la semiología. Todo el mundo hablaba constantemente de la lengua, el discurso, los signos, los códigos. Había una preocupación importante por conocer de cerca la semiótica, patente en la fascinación que ejercía sobre sociólogos de la talla de Jesús Ibáñez y Carlos Moya. Las modas son buenas porque permiten profundizar en ellas, pero son ondas efimeras, que van y que vienen y desaparecen como la espuma (Lozano, comunicación personal, 24 abril 2016).

Esa popularidad, desde luego, acrecentará el atractivo de la

comunicación. 
semiótica en una circunstancia histórica especial. El tránsito a la democracia, la libertad de prensa casi irrestricta y la expansión de la cultura de masas, generaban una fuerte demanda de herramientas conceptuales que facilitaran la comprensión de los nuevos discursos y los novedosos plexos de sentido. Así lo recuerda Moragas:

Cuando nació la semiótica, nuestra principal preocupación era saber interpretar; y además, en el contexto político que estábamos, saber interpretar entre líneas (...) la introducción de la sociosemiótica, diríamos, como un aire nuevo en el nuevo reclamo de la democracia (Moragas, 11 diciembre 2016).

\subsection{Iniciación en la disciplina}

En el plano personal, la mejor prueba del magnetismo que ejercía ese cuerpo de saberes es la disposición de los entrevistados por conocerlo de primera mano en una suerte de viaje iniciático. Tal es así que, a principios de los años 70, Moragas, un joven profesor de periodismo, marcha a Francia a verse con Edgar Morin, Violet Leduc, Cristian Metz y Abraham Moles, luminarias de los estudios en comunicación, y posteriormente a Urbino y Bolonia, donde contactará con Eco. A Talens, un flamante filólogo atento a las novedades intelectuales del extranjero, le sedujo la revista Semiotica de la IASS, y sobre todo, la lectura en 1973 de La estructura del texto artístico de Lotman (1982), fascinación que le movió a asistir a los encuentros en Urbino, un foco de la semiótica europea. Más tarde, Pérez Tornero, también de formación filológica, incitado por un seminario dictado en 1977 en la UAB por el propio Eco, viaja a París a estudiar con Greimas.

La bibliografía francesa, incluidos los análisis fílmicos de la revista Cahiers du Cinema, sumada a la lectura de Gillo Dorfles y Eco, seducen a Lozano, un joven licenciado en historia, y en 1976 irá a Bolonia con Cristina Peña Marín, a estudiar con Eco. En los años '80, Lacalle repetirá ese itinerario y, antes de regresar a Barcelona, ejercerá la docencia en la universidad italiana. Zunzunegui es el único que no realiza un desplazamiento de esas características. Su formación en la semiótica es de tipo autodidacta; lee tempranamente, y con gran interés, Obra Abierta (1966) de Eco y otras obras del mismo autor, aunque su conversión a la semiótica se produce años más tarde, con el estudio de la obra de Greimas, 
a quien conocerá personalmente.

Esas lecturas y esos viajes definirán las escuelas que estos jóvenes profesores difundirán desde los agrupamientos de los que formarán parte. El primero, y el más nutrido, se constituye en la UAB con la participación de Moragas, Lluis Bassets, Teresa Velázquez García, Helena Usandizaga, Pérez Tornero, Lorenzo Vilches, Miquel Rodrigo Alsina y Lacalle. Allí Moragas (1976) defiende la primera tesis doctoral en semiótica de la comunicación de masas, y allí se aplica la mirada semiótica a la publicidad (Pérez Tornero, 1982); la radiofonía (Bassets, 1983); la viñeta (Velázquez García y Ruiz Collantes, 1983); la fotografía (Vilches, 1983); el discurso periodístico (Rodrigo Alsina, 1986); o la televisión (Lacalle, 1992), entre otros objetos de estudio.

Poco más tarde se forma un grupo en la facultad de Ciencias de la Información (UCM), integrado por Lozano, Peña Marín y Abril, centrado en el análisis del discurso (Lozano et al, 1982; Peña Marín, 1980), el discurso histórico (Lozano, 1987), la moda (Abril, 1984); teoría de la información (Abril, 1999). En Valencia, Talens divide sus energías entre el cine (1986), el grafitti (1997) y el rock (Puig y Talens, 1997). Por último, en el País Vasco, Zunzunegui se centrará en el cine (Zunzunegui, 1985) y la semiótica de la imagen (Zunzunegui, 1989).

A ello hay que añadir las valiosas aportaciones de las asociaciones Andaluza de Semiótica y Gallega de Semiótica, centradas principalmente en semiótica literaria.

\subsection{La inserción académica}

Como hemos dicho, la institucionalización de la semiótica se expresó en dos planos académicos: el asociativo y el universitario. En relación al primero, los entrevistados señalan que el congreso de Semiótica e Hispanismo celebrado en Madrid, en 1983 -donde se fundó la AES, por iniciativa de Romera Castillo, celebrando su primer congreso en Toledo, al año siguiente-, separan la "prehistoria" de la "historia" de la semiótica autóctona. La entidad sirve de referencia y garantiza un mínimo de continuidad a quienes cultivan la disciplina, brindando con sus congresos cuatrienales un foro de intercambio y encuentro, y la vez estimulando otros agrupamientos y actividades de todo tipo (seminarios, simposios, cursos de verano, etc.). A mediados de los años ' 80 , ya se distinguen los 
tres carriles por los que discurrirá la semiótica española:

uno es la semiótica y la comunicación de masas, que con la creación de las facultades (de periodismo, n. d. a.) tiene un cierto progreso, aunque finalmente se estanca; otro es la semiótica lógica, más en el campo de la filosofía; y luego la semiótica del discurso literario (Moragas, comunicación personal, 11 diciembre 2016).

Detengámonos en la conexión que establece Moragas entre el primer carril y la proliferación de facultades de ciencias de la información en España a partir de mediados de los años '70. Nacidas con la idea de equipar a sus estudiantes con conocimientos de humanidades y ciencias sociales ausentes en las eminentemente prácticas escuelas de periodismo, su puesta en marcha conllevará la dotación de plazas docentes y oportunidades para la enseñanza de la semiótica. Lacalle aprecia en este anclaje institucional un rasgo distintivo de la recepción de la disciplina:

Lo que pasa con la semiótica en España, y en buena parte en Latinoamérica, a diferencia de Francia e Italia, los otros grandes focos de la semiótica (aquella, n. d. a.) encuentra su espacio en el ámbito de la información-comunicación, no en las facultades o en las licenciaturas de filosofía como en Italia, ni en las de literatura o de sociología como en Francia (Lacalle, comunicación personal, 24 noviembre 2016).

El primer ejemplo lo ofrece el propio centro donde Moragas se desempeña: la facultad de Ciencias de la Información de la UAB; y, más concretamente, la licenciatura de Periodismo:

Durante mi decanato de 1978 a 1980, la semiótica entra a formar parte como asignatura especifica en los planes de estudio de dicha titulación. Hay una revisión de planes de estudios y se introduce Semiótica de la Comunicación de Masas en el $4^{\circ}$ curso, que imparto con Lluis Bassets (Moragas, comunicación personal, 11 diciembre 2016).

El ejemplo barcelonés no tuvo eco inmediato en las demás 
universidades. Más bien ocurrió al contrario, pues los entrevistados subrayan las dificultades que encontraron para introducir la disciplina en las carreras ligadas a la comunicación: Periodismo, Comunicación Audiovisual, Publicidad y Relaciones Públicas.

En la UPV, informa Zunzunegui, nunca hubo una asignatura específica de semiótica, sino contenidos afines impartidos en varias asignaturas. "En mi facultad, la semiótica se introdujo muy tardíamente, y ha costado mucho", reconoce Lozano. Se queja de que, durante largo tiempo, en Ciencias de Información de la Complutense hubo que conformarse con impartir enfoques semióticos de forma transversal en las asignaturas “Teoría de la Información" y "Metodología de la investigación". Recién en 1987, la licenciatura de Periodismo acoge una asignatura específica, la optativa "Semiótica de la moda", y en 1995, la optativa "Semiótica de la comunicación de masas".

Talens, que daba semiótica dentro de la asignatura "Teoría Literaria" en la facultad de Filología (UV) -como hiciera también el profesor Romera-, atribuye las dificultades mentadas por Lozano al control de las facultades de comunicación por gestores poco proclives a potenciar saberes cuya utilidad profesional no veían: "Si antes había estudios de periodismo en un 90 por ciento controlados por la Iglesia, las ciencias de la información estaban controlada por periodistas, no necesariamente académicos" (Talens, comunicación personal, 9 marzo 2016).

A su modo de ver, ese sesgo profesionalista hostil a la teoría trabó la ampliación de los incipientes contenidos semióticos en los planes de estudio, en cuyo diseño los semiólogos incidieron poco. Esa resistencia se volvió a poner de manifiesto más tarde, cuando la apertura de las licenciaturas en comunicación audiovisual creó un espacio para la semiótica visual que no fue aprovechado: "La especialidad en Comunicación Audiovisual empieza en los años 90, y no creo que la semiótica haya tenido en ella un papel fundamental, aunque debería haberlo tenido" (Talens, comunicación personal, 9 marzo 2016).

Con la reforma de Bolonia, la posición de la disciplina en los estudios de grado experimentó altibajos. En la Complutense, explica Lozano, la optativa "Semiótica de la comunicación de masas" se torna de cursado obligatorio en el grado de Periodismo. El mismo estatuto, apunta Talens, adquieren "Semiótica de la Comunicación" en el Grado de Comunicación Audiovisual de la UV, y "Semiótica de la Comunicación 
de Masas" y "Semiótica de la publicidad y el consumo" en el Grado de Publicidad y Relaciones Públicas de la Universidad de Alicante.

En cambio, en la UAB se nota un retroceso. La optativa "Semiótica de la comunicación de masas" de la licenciatura de Comunicación Audiovisual pasa a llamarse "Semiótica de la Comunicación" en el Grado de Comunicación Audiovisual, pero se acorta de anual a semestral:

El retroceso a partir de Bolonia en la mayor parte de las facultades ha sido flagrante y, en particular, en mi universidad. La semiótica se ha convertido en una asignatura optativa de cuarto año, una asignatura que - pese al ingente esfuerzo del equipo docente por construir algo divulgativo - es una disciplina compleja y hay muchos estudiantes de cuarto año que ya no quieren complicarse la vida de esa manera. Por otra parte, a punto de terminar la licenciatura tienen prácticas, tienen Erasmus, les resulta dificil a nivel mental - por lo menos de conjugar - y de situar la teoría en ese momento de la carrera (Lacalle, comunicación personal, 24 noviembre 2016).

En la UPV no hubo novedades, comenta Zunzunegui, y la semiótica continúa siendo un enfoque dentro de las asignaturas "Historia del cine" y "Narrativa Audiovisual" en el Grado de Comunicación Audiovisual; un enfoque cuyo dictado, enfatiza, queda al arbitrio del docente.

En ocasiones, los postgrados impulsados por Bolonia abren resquicios. Refiere Lozano que en su facultad el máster en Investigación en Periodismo: Discurso y Comunicación acoge la asignatura "Semiótica de la Cultura y Semiótica de la Moda”, y el máster en Análisis Sociocultural del Conocimiento y la Comunicación hace lo propio con "Análisis semiótico de textos y procesos culturales". Pero en la UAB, indica Lacalle, no ha ocurrido nada parecido en ningún máster.

Abundando en lo apuntado por Zunzunegui, Talens sostiene que la suerte de los contenidos semióticos ha dependido de los profesores que pugnaban por su continuidad en los planes de estudio. Y si estos bajaban la guardia, dichos contenidos no tardaban en ser eliminados por sus colegas:

Cuando me fui a Ginebra era director de departamento en Valencia [...] lo primero que hicieron fue quitar del plan de estudio la asignatura de Análisis Textual que yo había puesto. En cuanto 
regresé a Valencia, la volví a instalar, esta vez en el máster. Me pareció sintomático que de la cantidad de cosas que podian haber eliminado la única que quitaron fuera la semiótica. Está claro por dónde iban los tiros (Talens, comunicación personal, 9 marzo 2016).

Cuarenta años después de la introducción de la primera asignatura específica de semiótica en una universidad española, el balance de Pérez Tornero es taxativo: "no se incorporó bien a los programas de estudio del área de comunicación; ahora hay mucha demanda de comunicación política [...] la semiótica hubiera podido ocupar ese terreno" (Pérez Tornero, comunicación personal, 11 diciembre, 2016).

Un dato significativo es que ninguno de los entrevistados obtuvo posiciones académicas asociadas a un perfil nominalmente semiótico: todos accedieron a cátedras de periodismo, literatura, teoría de la información o comunicación audiovisual. Una realidad institucional que viene a cuenta de las desventajas que, al decir de Pérez Tornero, un perfil de ese tipo podía entrañar de cara a la consolidación profesional:

En España, para conseguir las cátedras algunos tuvieron que hacer apostasía de la semiótica. Tuvieron que hacer apostasía, renunciar. [...] yo conozco varios. Si ibas de semiótico no ganabas la plaza, y punto. Y era así de claro (Pérez Tornero, comunicación personal, 11 diciembre 2016).

A tenor de lo expuesto por se aprecia que el impulso inicial registrado a principios de los ' 80 favoreció la penetración académica de la semiótica, aunque su arraigo no estuvo exento de obstáculos y retrocesos. Es más, las posiciones ganadas tampoco están seguras, pues su pervivencia en los planes de estudios depende en sumo grado del empeño de los semiólogos.

\subsection{El reflujo}

Los entrevistados apuntan que los modestos avances de la subdisciplina en el plano curricular se dieron en un contexto de progresiva disipación del interés que el profesorado y la intelectualidad española sentían por la semiótica. El desapego ya era palpable a finales de los años ' 80 , y se manifiesta en el alejamiento de unos cuantos semiólogos, que se 
dedican a otras profesiones o campos de estudios (tal el caso de Bassets, que se decanta por el periodismo; el de Vilches, orientado hacia la industria televisiva; o el de Juaristi, que se vuelca a la literatura):

A finales de los '80, ya te empiezan a decir los colegas: "Oye, ¿pero eso de la semiótica no es una cosa que está pasada de moda?". En España, la academia es como las cotizaciones de la Bolsa: suben $y$ bajan, y ahora se pone de moda esto, y ahora lo otro, y ahora hay que hacer esto y ahora hay que hacer lo otro (Zunzunegui, comunicación personal, 15 noviembre 2016).

Y coinciden en que en modo alguno se trataba de un fenómeno local. Observa Talens que en la propia Italia, "una figura como Casetti empezó a ver que la semiótica no tenía muy buena prensa en la universidad y empezó a irse a otros territorios". Lozano se expresa en sentido parecido:

El día en que murió Roland Barthes (1980, $n$. de. a.) muchísima gente abandonó el barco. Con él desapareció la cátedra de Semiologie Littéraire en el College de Francia. Julia Kristeva, autora de textos admirables de semiótica, hoy ejerce de psicoanalista católica. $Y$ Todorov a veces se presenta como semiólogo, como historiador del pensamiento, o filósofo (Lozano, comunicación personal, 24 abril 2016).

Importa resaltar que dos de los entrevistados participan de ese distanciamiento. Pérez Tornero es uno de ellos:

Yo estaba muy mal en la facultad en el sentido de ser semiótico más no tener la plaza fija... pues, me ofrecieron dirigir el departamento de TVE de Educativos porque habia hecho cosas de prensa, de televisión y tal. Y me tomé un respiro y me fui a Madrid (Pérez Tornero, comunicación personal, 11 diciembre 2016).

En línea con las percepciones de Pérez Tornero, Lacalle advierte que en el abandono de la semiótica por parte de algunos colegas pesaron factores como la estabilidad profesional, pues estos percibían que apostar por esa especialización les cerraría las puertas: 
Se empiezan a, por una parte, a autoconsiderar (semiólogos, n. d. a.) pero, por otra parte, temiendo hasta cierto punto ser expelidos a los bordes del sistema, la abandonan. Para mí es descorazonador en más de una ocasión - en contextos diversos: congresos, tribunales de tesis, etc.- me he encontrado con personas que (me decían, $\mathrm{n}$. d. a.) “-Ah, ipero todavía sigues con eso de la semiótica?” [...] y hay quien incluso de manera anecdótica cuenta: "Yo empecé con la semiótica pero luego lo dejé porque vi que por ahí no llegaba a ninguna parte" (Lacalle, comunicación personal, 24 noviembre 2016).

En el alejamiento de Moragas no intervinieron apremios relativos a la carrera docente, pues se trató de una reorientación de su perfil académicoinvestigador en respuesta a demandas externas:

Ha habido una transformación enorme en el sector de las comunicaciones y nuevos retos [...] llegados al 84, a la semiótica no le piden lo mismo que antes, que era un análisis crítico de la cultura, le están pidiendo soluciones para democratizar los medios. $Y$ nosotros, la gente que nos dedicamos a este oficio, con el input que nos va llegando de la sociedad [...] se van sucediendo las preguntas políticas. Es bastante evidente que hay una sobrecarga en la demanda hacia las políticas de comunicación [...] y yo mismo vario un poco mi trayectoria, el trabajo de análisis semiótico lo veo más como economía política de los medios intentando responder a las demandas sociales que se están acelerando (Moragas, comunicación personal, 11 diciembre 2016).

En las palabras de Moragas y Pérez Tornero se aprecia que la evolución de la comunicación de masas en España les planteó retos más tentadores que la dedicación exclusiva a la semiótica académica.

Otro factor que conspiraba contra la consolidación de la subdisciplina era la debilidad de la comunidad de semiólogos especializados en los mass media. Varios entrevistados señalan que, como se veía en los congresos de la AES, ellos representaban una minoría frente a una mayoría dedicada a los análisis semiótico-literarios; encima, a esa minoría le faltaba 
cohesión interna:

Mi objetivo hubiera sido crear una comunidad cientifica en torno a los problemas de comunicación en el sentido amplio de la semiótica. [...] Eso se ha ido diluyendo, no existe. Y luego, aqui éramos pocos y nos peleamos; el grupo de Madrid contra el grupo de Barcelona, el grupo de Barcelona contra el grupo de Madrid, unos contra otros, y yo dije: "Mira ya, paso de la semiótica (Pérez Tornero, comunicación personal, 11 diciembre 2016).

Lozano hace hincapié en el efecto debilitador que los abandonos tuvieron en la emergente comunidad de semiólogos, pero los atribuye a causas distintas a la estabilidad profesional:

Masa crítica ha habido mucha, pero también mucho abandono. Y si me pongo severo, diré que la razón del abandono es muy sencilla: hay ciertos conocimientos que no exigen una formación permanente, y en cambio, la semiótica sí. Muy pocos están dispuestos a dedicar dos años al estudio profundo de Greimas. Y entonces ha habido abandono (Lozano, comunicación personal, 24 abril 2016).

Sin embargo, ese alto nivel de exigencia que Lozano juzga fundamental para el ejercicio y la enseñanza de la semiótica tuvo un efecto contraproducente, según apuntan otros entrevistados:

La palabra semiótico ha quedado un poco hiperléxicográfico. $O$ sea, demasiado léxico, demasiada oscuridad. La semiótica se ha refugiado en un lenguaje esotérico [...] Ha asustado a la gente. Ha asustado la jerga. Como si hubiese tenido la necesidad de una jerga propia para autodefinirse en el campo de las ciencias humanas (Moragas, comunicación personal, 11 diciembre 2016).

En un sentido parecido se manifiesta Lacalle al atribuir al excesivo cerramiento de la disciplina en sí misma el distanciamiento de sus compañeros de viaje:

La semiótica estructuralista complicó de tal manera el metalenguaje 
que, poco a poco, nos fuimos quedando quienes realmente queríamos especializarnos con una actitud, a veces negativa respecto a las personas de fuera [...] creo que esto descorazonó mucho a otros investigadores que no querían dedicar su tiempo total a la semiótica sino que la consideraban un instrumento de reflexión teórico/metodológica muy interesante para completar perspectivas sociológicas, narratológicas y literarias, mediáticas, etc. Este tipo de discípulo semiótico se fue retirando [...] ha habido en algunos sectores cierta arrogancia intelectual que ha podido desanimar, o incluso alejar, a investigadores de otras disciplinas (Lacalle, comunicación personal, 24 noviembre 2016).

En una mirada retrospectiva, Talens resume el itinerario recorrido del siguiente modo:

La historia de la semiótica española ha sido la historia de una dificultad. Siempre hubo poca masa crítica. Éramos núcleos muy pequeñitos y muy aislados. Los escasos grupos se movían en estratos diferentes y no dialogaban entre sí, no interesaba. [...] la semiótica goza de muy mala salud y muy mala fama en España (Talens, comunicación personal, 9 marzo 2016).

\subsection{Diagnóstico y pronósticos}

En cuanto a las perspectivas de la especialidad, los entrevistados se muestran pesimistas. Sienten en su entorno una fuerte resistencia a la semiótica, que algunos ligan al avance de los paradigmas positivistas y las metodologías cuantitativas:

Hemos sido objeto de un rechazo reaccionario, conservador, de un intento de mantener el status quo de ciertos conocimientos, de métodos como el análisis de contenido. Eso da el confort heurístico de dar un dato; por eso hoy estamos en la época más necia del mundo, la del Big Data, que consiste en tener más datos. En el extranjero se pasó del análisis de contenido a la semiótica, ya desde los años 60. Aqui ha ocurrido al revés: se pasó de la semiótica al análisis de contenido, en una involución enorme (Lozano, comunicación 
personal, 24 abril 2016).

Talens comparte la percepción de su colega respecto del ambiente adverso generado por el avance arrollador de los paradigmas cuantitativos:

En mi universidad y en otras en las que he sido miembro de tribunales de tesis doctorales hay una reticencia enorme a la semiótica, incluso en las carreras de periodismo. En algunos sitios si dices "semiótica" es como si mentaras al diablo. En Valencia, colegas con los me llevo muy bien siempre me dicen: “iDemasiada semiótica!”. Son más partidarios de los estudios cuantitativos (Talens, comunicación personal, 9 marzo 2016).

Y Moragas advierte que no se puede ignorar el peso que las demandas de los estudiantes han tenido en la pérdida de predicamento de la semiótica en las carreras de comunicación:

Hoy en la formación universitaria hay una más grande preocupación para saber hacer que por saber interpretar... Cuando nació la semiótica, nuestra principal preocupación era saber interpretar, y, en el contexto político que estábamos, saber interpretar entre líneas [...] esta pasión por el saber interpretar me parece que hoy está un poco a la baja; porque más bien son intentos de saber hacer y saber resolver, saber aplicar (Moragas, comunicación personal, 11 diciembre 2016).

Lacalle encuentra grandes similitudes entre las poco halagüeñas perspectivas de la semiótica local y la situación en los países que fueron la referencia internacional en el campo: "(en España) va a ir a peor porque ya ha ido a peor en Italia y en Francia. En las facultades - por ejemplo, París, el gran foco de donde parte todo- está, como semiótica, de manera oficial en vías de extinción" (Lacalle, comunicación personal, 24 noviembre 2016). A su entender, el declive tiene mucho que ver con el pasaje del liderazgo académico a los países anglosajonas, que a través de sus prestigiosas publicaciones fijan los estándares de calidad y los paradigmas teóricos juzgados aceptables: "Como la semiótica no terminó de penetrar en el ámbito anglosajón, eso ha limitado sus posibilidades, 
por ejemplo, de expansión de revistas académicas con los criterios de los índices internacionales, etc." (Lacalle, comunicación personal, 24 noviembre 2016).

Retomando lo antes apuntado por Pérez Tornero, Talens constata que a la semiótica le han comido el terreno otras especialidades, y eso le vuelve pesimista:

La semiótica debería ser una asignatura central. Lo que pasa que los comunicólogos están cayendo en manos de gente de marketing y estudios de mercado, de sociología de la comunicación, pero no de análisis del discurso. Que sea eso lo que se potencie desde arriba es un mal asunto [...] Veo mal futuro (Talens, comunicación personal, 9 marzo 2016).

Menos dramático se muestra Moragas, posiblemente porque ya no se considera miembro de la comunidad de semiólogos. En su visión de futuro, la semiótica perdura como una corriente más dentro del núcleo duro teórico de las ciencias de la comunicación, diluida en el seno de una "post disciplina":

Me gusta la idea de post disciplina. Una post disciplina no significa que no reconozcamos todo lo anterior; hay cantidad de trabajos de clásicos de la semiología que siguen siendo fundamentales para interpretar cualquier signo contemporáneo (Moragas, comunicación personal, 11 diciembre 2016).

Con todo, para Lozano el balance no es enteramente negativo. Destaca que la semiótica ha impregnado la cultura, la doxa y el vocabulario de expertos y legos con una especie de vulgata:

Ocurre como con aquel personaje de Moliere que hablaba en prosa sin saberlo: hay muchisimos elementos del habla común que hacen de la semiótica un conocimiento incorporado, sin saberlo, pero la perspectiva semiótica está. Insisto en una frase de Eco: "la gente habla de código genético sin haber leído un solo libro de semiótica". Ha habido una presencia muy fuerte e irreversible de la semiótica en los estudios de comunicación de masas, que no tiene fecha, 
como nadie sabe quién es el inventor del IPad o de conocimientos más o menos colectivos que no tienen nombres propios (Lozano, comunicación personal, 24 abril 2016).

\section{CONCLUSIONES}

El análisis de las entrevistas ha permitido reconstruir la historia de la semiótica de la comunicación de masas en el medio académico español desde el punto de vista de algunos de sus protagonistas destacados. La historia en otros ámbitos se ha realizado asimismo.

Las reminiscencias de los semiólogos confirman lo ya sabido respecto del temprano eco de la semiótica en España, y aportan datos novedosos relativos a las circunstancias de lugar y tiempo de su recepción: la Barcelona de la segunda mitad de los años '60, la vía de entrada de la obra de Eco y el escenario de los primeros escarceos académicos con ese saber todavía foráneo. Nos informa asimismo de cómo se fue aclimatando gracias al esfuerzo de los jóvenes graduados que se desplazaron a Francia e Italia para, a su regreso, introducir en los centros académicos donde comenzarían sus carreras lo aprendido en las escuelas de Greimas y de Eco.

Con sus evocaciones no exentas de nostalgia, los entrevistados califican los años "70 de "época dorada" de la disciplina en el plano internacional y en el doméstico. Su prestigio en un contexto de apertura y modernización de la sociedad y la universidad española, propició la eclosión de una comunidad vernácula de semiólogos. En los años ' 80 surge una asociación nacional y varias regionales, se publican trabajos sobre cine, fotografía, discurso político, publicidad, discurso histórico o narración televisiva, y la enseñanza de la semiótica se abre paso en las carreras ligadas a la comunicación.

Pero el impulso inicial se fue debilitando a medida que la disciplina perdía su aura fuera y dentro de nuestras fronteras, y su consolidación universitaria chocaba con resistencias. Las cortapisas se evidenciaban en la dificultad para introducir en los planes de estudio asignaturas consagradas al saber semiótico y en las trabas al desarrollo de carreras académicas como "semiólogos". A esto se sumó el alejamiento de especialistas de la primera hora, produciéndose una pérdida de "masa crítica" intelectual.

Pese a que la semiótica ha mantenido cierta presencia a través de 
los sucesivos cambios de los planes de estudio, el diagnóstico sombrío de la situación actual es unánime. De su retroceso los entrevistados culpan a factores endógenos como el elitismo del propio colectivo, los conflictos intestinos y la creciente complejidad de su marco categorial; o exógenos como la supremacía de los enfoques y métodos empírico-cuantitativos y la vertiginosa mutación del sistema mediático que amenaza con dejar obsoletos los paradigmas tradicionales. La falta de un relevo generacional empeora las perspectivas y hace temer a los semiólogos por la supervivencia de su disciplina.

Ciertamente, el relato expuesto es un compuesto de percepciones irreductiblemente subjetivas; no obstante, estas manifestaciones nada complacientes vienen a enriquecer una panorámica que quedaría incompleta de limitarse a los datos objetivos de los repertorios bibliográficos, los programas de estudio o la relación de congresos y seminarios. Desde luego, dicha panorámica lucirá más completa si ulteriores estudios recogen las vivencias de los demás componentes de la comunidad semiótica española: los especialistas consagrados a los estudios teatrales y literarios.

Queda, por último, apuntar que en más de un aspecto el itinerario reconstruido guarda parecido con la suerte corrida por otras disciplinas y corrientes humanísticas (reparemos en el auge y olvido del estructuralismo). Comprender que las vicisitudes referidas en estas páginas no son privativas de la semiótica debería apercibirnos de que no es este el único saber en peligro de extinción ante el inminente acortamiento de los grados (el modelo " $3+2$ ") en beneficio de posgrados enfocados en el mercado laboral, y promover una reflexión sobre cómo se ha llegado a esta situación y cómo afrontarla.

\section{REFERENCIAS BIBLIOGRÁFICAS}

ABRIL, G. (1976). Signo y significación. Madrid: Pablo del Río. (1999). Teoría general de la información; datos, relatos y ritos. Madrid: Cátedra.

BARTHES, R. (1974). "Retórica de la Imagen". En La semiología, AA.VV., 127-140, Buenos Aires: Tiempo Contemporáneo. (1980). Mitologías. México: Siglo XXI.

BASSETS, L. (1983). "Quatre apunts sobre semiótica radiofónica". 
Anàlisi 7-8, 179-191.

BOBES NAVES, M. C. (1973). La semiótica como teoría lingüística. Madrid: Gredos.

BOURDIEU, P. (1989). "La ilusión biográfica”. Historia y Fuente Oral 2, 27-33.

ECO, U. (1978). Il superuomo de massa. Milano: Bompiani.

(1983). TV: la transparenza perduta. Milano: Bompiani.

GUBERN, R. (1974). Mensajes icónicos en la cultura de masas. Barcelona: Lumen.

(1977). Comunicación y cultura de masas. Barcelona: Península.

LACALLE, C. (1992). La serialidad en la información televisiva: los telediarios. Barcelona: Universitat Autònoma de Barcelona.

LOTMAN, Y. (1982). La estructura del texto artístico. Madrid: Fundamentos.

LOZANO, J.; PEÑA MARIN, C. y ABRIL, G. (1982). Análisis del discurso. Hacia una semiótica de la interacción textual. Madrid: Cátedra.

LOZANO, J. (1987). El discurso histórico. Madrid: Alianza Editorial.

MARIEZKURRIELA ITURMENDI, D. (2008). "La historia oral como método de la investigación histórica". Gerónimo de Ustariz 2324, 227-233.

MORAGAS, M. (1976). Semiótica y comunicación de masas. Barcelona: Península.

PAZ GAGO, J. M. y COUTO CANTERO, P. (1999). "La semiótica en Galicia: la Asociación Gallega de Semiótica”. Signa 8, 125-150 (también en http://www.cervantesvirtual.com/portales/signa/obravisor/signa-revista-de-la-asociacion-espanola-de-semiotica--3/ html/dcd93078-2dc6-11e2-b417-000475f5bda5_26.html\#I_28_ [02/04/2019]).

PEÑA MARIN, C. (1980). "Una aproximación intertextual al análisis del discurso". REIS 12, 141-157.

PÉREZ-TORNERO, J. M. (1982). La semiótica de la publicidad. Barcelona: Mitre.

POZUELO-YVANCOS, J. M. (1999). "La Asociación Española de Semiótica: crónica de una evolución científica". Signa 8, 53-69 (también en http://www.cervantesvirtual.com/obra-visor/signa-revistade-la-asociacion-espanola-de-semiotica--3/html/dcd93078-2dc6- 


\section{1e2-b417-000475f5bda5_20.html\#I_10_[02/04/2019]).}

PUIG, L. y TALENS, J. (1997). "Rock cuerpo y simulacro". Zehar 35, 20-25.

RÍOS, F. J. (2015). "La Asociación Española de Semiótica (AES). Treinta años de investigaciones semióticas en España". Signa 24, 23-36 (también en

http://revistas.uned.es/index.php/signa/article/view/14708/13123 [02/04/2019]).

RODRIGO ALSINA, M. (1986). "El uso de la ilusión referencial en el discurso periodístico informativo". En Investigaciones semióticas I, AA.VV, 463-472, Madrid: CSIC.

ROMERA CASTILLO, J. (1988). Semiótica literaria y teatral en España. Kassel: Reichenberger.

(1991). "Introducción a la semiótica (notas bibliográficas)". En Introducción a la semiótica: actas del curso de Introducción a la semiótica, A. Sánchez Trigueros y J. R. Valles Calatrava (eds.), 3-11. Almería: Instituto de Estudios Almeriense.

(2015). "La Asociación Española de Semiótica impulsora de la modernización del panorama científico". Signa 24, 1322 (también en http://revistas.uned.es/index.php/signa/article/ view/14714 [02/04/2019]).

(2016). "La revista Signa: 25 años de andadura científica". Signa 25, 13-76 (también en http://www.cervantesvirtual.com/obra/revistasigna/; http://www2.uned.es/centro-investigacion-SELITEN@T/pdf/ signa/SIGNA_25_NUMEROS.pdf[20/02/2019]).

TALENS, J. (1986). El ojo tachado: lectura de "Un chien andalou” de Luis Buñuel. Madrid: Cátedra.

(1997). "Los muros para el que los trabaja". En Los muros tienen la palabra: materiales para una historia de los graffiti, F. Gimeno Bay y M. L. Mandigorra Llavata (eds.), 7-10. Valencia: Universitat de Valencia.

URRUTIA, J. (1975). Sistemas de comunicación. Barcelona: Planeta.

VÁZQUEZ MEDEL, M. Á. (1999). "La Asociación Andaluza de Semiótica". Signa 8, 87-101 (también en http://www. cervantesvirtual.com/portales/signa/obra-visor/signa-revista-dela-asociacion-espanola-de-semiotica--3/html/dcd93078-2dc611e2-b417-000475f5bda5_23.html\#I_19_[02/04/2019]). 
VELÁZQUEZ GARCÍA, T. y LACALLE, C. (1999). "La semiótica en Cataluña". Signa 8, 117-123 (también en http://www. cervantesvirtual.com/portales/signa/obra-visor/signa-revista-dela-asociacion-espanola-de-semiotica--3/html/dcd93078-2dc611e2-b417-000475f5bda5_25.html\#I_25_[02/04/2019]).

VELÁZQUEZ GARCÍA, T. y RŪIZ COLLANTES, X. (1983). “Análisis discursivo de la serie de Peridis sobre el viaje de Felipe González a Marruecos". En Teoría semiótica: lenguajes y textos hispánicos, M. Á. Garrido Gallardo (ed.), vol. 1, 785-804. Madrid: CSIC.

VERÓN, E. (1993). Construir el acontecimiento. Barcelona: Gedisa.

VILCHES, L. (1983). "El rol del lector en la fotografía". Anàlisi 7-8, 161177.

YLLERA, A. (1979). "Les recherches sémiologiques en Espagne”. En Le champ sémiologique: perspectives internationales, A. Helbo (ed.), 1-42. Bruxelles: Complexe.

ZUNZUNEGUI, S. (1985). "Imagen, documental, ficción". Revista de Ciencias de la Información 2, 53-62.

(1989). Pensar la imagen. Madrid: Cátedra.

Recibido el 11 de enero de 2019.

Aceptado el 13 de marzo de 2019. 